\title{
Coagulation screening tests in high risk neonates: a prospective cohort study
}

\author{
B K Schmidt, P Vegh, M Andrew, M Johnston
}

\begin{abstract}
Forty seven infants in a prospective cohort of 170 high risk neonates without signs of overt bleeding had abnormal coagulation screening tests within 36 hours of birth. Early thrombocytopenia was a better predictor of prolonged prothrombin times and hypofibrinogenaemia than very low birth weight, fetal growth retardation, or poor five minute Apgar scores.
\end{abstract}

(Arch Dis Child 1992;67:1196-7)

Although the occurrence of neonatal thrombocytopenia has been studied, ${ }^{1}$ little is known about the prevalence of plasma clotting defects in unselected high risk infants. We therefore performed coagulation screening tests within 36 hours of birth in a prospective cohort of critically ill neonates. We also examined whether abnormalities of plasma coagulation can be predicted by early low platelet counts and several other generally accepted risk factors such as very low birth weight, fetal growth retardation, and low five minute Apgar scores.

\section{Subjects and methods}

SUBJECTS

The study was conducted in the neonatal intensive care unit at McMaster University between April 1989 and March 1990. All infants admitted to the unit were eligible for enrolment if the following two criteria were met: (i) postnatal age less than 36 hours and (ii) indwelling arterial catheter with undampened pressure tracing. The latter ensured recruitment of the group of patients in the nursery who were most ill and also allowed artifact free blood sampling. The study was approved by the Institutional Review Board for research involving human subjects.

The decision to place an arterial catheter for monitoring purposes was made by the attending medical staff without reference to this study. Patency of the catheter was maintained with an infusion of $5 \%$ dextrose containing $1 \mathrm{U} / \mathrm{ml}$ heparin at a rate of $1 \mathrm{ml} /$ hour. All flushing solutions were heparin free. Apart from the administration of $24 \mathrm{U} /$ day heparin, no study patient received anticoagulant or thrombolytic drugs. Vitamin $\mathrm{K}(0.5-1.0 \mathrm{mg})$ was given parenterally at birth to all infants. Administration of blood products during the six hours preceding the study sample was recorded in all subjects.

COAGULATION ASSAYS

Blood samples $(0.9 \mathrm{ml})$ for coagulation studies were drawn once from the free flowing arterial catheter at the time of medically ordered sampling for complete blood counts. The prothrombin time was determined using human thromboplastin (Thromborel S, Behringwerke, Germany), and expressed as the international normalised ratio (INR)

$$
\left.\mathrm{INR}=\left(\frac{\text { Patient prothrombin time }}{\text { Control prothrombin time }}\right)\right)^{\mathrm{ISI}}
$$

where ISI is the international sensitivity index of the thromboplastin used in the local laboratory. ${ }^{2}$ Fibrinogen was measured using the method of Clauss. ${ }^{3}$ Platelet counts $<150 \times$ $10^{9} /$, fibrinogen concentrations $<1.5 \mathrm{~g} / \mathrm{l}$, and an INR $>1.5$ were considered abnormal in all study patients. ${ }^{14}$ Plasma samples were shown to be free of heparin contamination by protamine titration.

\section{ANALYSIS}

Observed frequencies and $95 \%$ confidence intervals of laboratory abnormalities were determined. Sensitivities, specificities, predictive values, and likelihood ratios were calculated to assess whether birth weight $<1500 \mathrm{~g}$, gestational age $<29$ weeks, five minute Apgar scores $<6$, and platelet counts $<150 \times 10^{9} / 1$ predicted abnormalities of plasma coagulation. ${ }^{5}$ As fetal growth retardation has been implicated as a risk factor of coagulopathy, the frequency of plasma coagulation defects was also examined in infants with birth weights below the tenth centile according to local population based standards. ${ }^{6}$

\section{Results}

STUDY INFANTS

During the 12 month study period, 191 infants fulfilled the two entry criteria, 177 of whom were entered into the study. Fourteen infants were either missed, or sampled after the first 36 hours of life. Seven blood samples were mislabelled or clotted. For ethical reasons the study protocol did not allow repeat samples to be drawn in these instances. Thus results are reported for 170 infants, or $89 \%$ of all potentially eligible infants. Table 1 summarises the

\begin{tabular}{lc} 
Table 1 Characteristics of the 170 infants studied \\
\hline Gestational age (weeks) & $29(24-41)^{*}$ \\
Birth weight (g) & $1380(600-5300)^{*}$ \\
Sex (male/female) & $93 / 77$ \\
In/outborn & $123 / 47$ \\
5 minute Apgar score & $7(2-10)^{*}$ \\
Postnatal age (hours) & $16 \cdot 6(0 \cdot 8-35 \cdot 5)^{*}$ \\
No with mechanical ventilation at & 144 \\
$\quad$ time of study & 14 \\
No with fresh frozen plasma infusions & 27 \\
$\quad$ before study & \\
\hline
\end{tabular}

*Values are median (minimum-, maximum) values. 
important characteristics of the study group. No infant had received blood products for clinical bleeding before the coagulation screen. Twenty seven infants were given up to 20 $\mathrm{ml} / \mathrm{kg}$ of fresh frozen plasma to improve hypovolaemia and hypotension during the six hours preceding the blood sample. Coagulation screening tests were entirely normal in $67 \%$ of patients who received plasma, compared with $63 \%$ of those who did not. Owing to the considerable overlap of the respective $95 \%$ confidence intervals $(46.0$ to $83.5 \%$ v 54.5 to $70.9 \%$ ), the effect of the plasma infusions on the test results was not considered important enough to justify stratification of subsequent analyses according to plasma use.

\section{ABNORMALITIES OF COAGULATION SCREENING TESTS}

Table 2 gives the observed frequencies of abnormal tests together with the $95 \%$ confidence intervals. Of the 47 infants with abnormal plasma coagulation, 18 patients had an increased INR, whereas 16 subjects had a low fibrinogen concentration. In a further 13 infants the two tests were abnormal. Among infants with an abnormal INR, the median value was $1 \cdot 7$ (maximum 3.1). Among infants with hypofibrinogenaemia the median fibrinogen concentration was $1.1 \mathrm{~g} / 1$ (minimum 0.3 $\mathrm{g} / \mathrm{l})$. The median platelet count in the 36 infants with thrombocytopenia was $118 \times 10^{9} / 1$ (minimum $36 \times 10^{9} / 1$ ).

\section{PREDICTION OF COAGULOPATHY}

Table 3 summarises the sensitivities, specificities, predictive values, and likelihood ratios for all examined risk factors of coagulopathy.

\section{Discussion}

Abnormalities of the coagulation system are reported to be common in ill neonates, yet little is known about the prevalence of plasma clotting defects in unselected high risk infants. In this prospective cohort study coagulation

Table 2 Abnormalities of coagulation screening tests in 170 high risk neonates

\begin{tabular}{lcc}
\hline Coagulation profile & No (\%) of occurrences & $\begin{array}{l}\text { 95\% Confidence } \\
\text { interval (\%) }\end{array}$ \\
\hline Normal & $108(64)$ & $55 \cdot 8$ to $70 \cdot 8$ \\
Coagulopathy & $26(15)$ & $10 \cdot 2$ to $21 \cdot 6$ \\
Coagulopathy and thrombocytopenia & $21(12)$ & $7 \cdot 8$ to $18 \cdot 3$ \\
Thrombocytopenia & $15(9)$ & $5 \cdot 0$ to $14 \cdot 1$ \\
\hline
\end{tabular}

Table 3 Prediction of coagulopathy

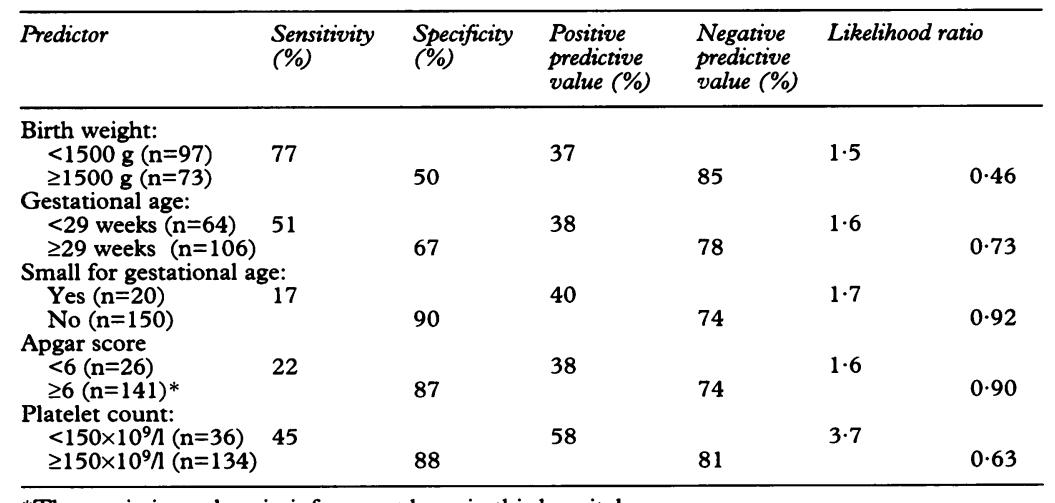

*Three missing values in infants not born in this hospital. screening tests were abnormal within the first 36 hours in about one third of all infants who were sick enough to require an indwelling arterial catheter for monitoring purposes. Abnormalities of plasma coagulation could not be predicted accurately by more readily known risk factors such as immaturity, fetal growth retardation, or poor Apgar scores. The strongest predictor of coagulopathy was thrombocytopenia. Infants with platelet counts $<150 \times 10^{9} / 1$ were 3.7 times more likely to have plasma coagulation defects than infants with normal platelet counts. Further, normal INR and fibrinogen values were less than half as likely to be found in infants weighing $<1500 \mathrm{~g}$ than in larger infants. Low birth weight, however, was not a strong predictor of abnormal plasma coagulation. Thus our data suggest that screening for coagulopathy will have the highest yield in infants with early thrombocytopenia whereas infants with birth weights $>1500 \mathrm{~g}$ are least likely to have laboratory evidence of coagulopathy.

The clinical significance of abnormal coagulation screening tests is currently uncertain. Although no infant was noted to bleed overtly at the time of the study, occult haemorrhage may well have contributed to the morbidity of these critically ill infants. This hypothesis is supported by previous descriptions of an association between abnormal coagulation tests and the presence and severity of intracranial haemorrhage in preterm infants. ${ }^{7-10}$ Trials of fresh frozen plasma or other preparations of coagulation factors in the prevention of intracranial haemorrhage have yielded conflicting results. ${ }^{11}$ Further studies are therefore required to determine whether sick newborn infants would benefit from the detection and correction of clinically inapparent clotting defects.

Presented at the annual meeting of the Society for Pediatric Research 1991 in New Orleans, LA, USA. Supported by a grant in aid from the Heart and Stroke Foundation of Ontario. Dr B K Schmidt is a Research Scholar of the Heart and Stroke Foundation of Canada. Dr M Andrew is a Career Investigator of the Heart and Stroke Foundation of Ontario.

1 Castle V, Andrew M, Kelton J, Giron D, Johnston M, Carter C. Frequency and mechanism of neonatal thrombocytopenia. $\mathcal{F}$ Pediatr 1986;108:749-55.

2 Hirsh J, Deykin D, Poller L. 'Therapeutic range' for oral anticoagulant therapy. Chest 1986;89:11S-5S.

3 Clauss A. Gerinnungsphysiologische Schnell-methode zur Bestimmung des Fibrinogens. Acta Haematol 1957;17: 237-46.

4 Hathaway W, Corrigan J. Report of scientific and standardization subcommittee on neonatal hemostasis. Normal coagulation data for fetuses and newborn infants. Thromb Haemostas 1991;65:323-5.

5 Sackett DL, Haynes RB, Tugwell P. The interpretation of diagnostic data. Clinical epidemiology. A basic science for clinical medicine. Boston: Little, Brown, 1985:59-138.

6 Arbuckle TE, Sherman GJ. An analysis of birth weight by gestational age in Canada. Can Med Assoc $\mathcal{F}$ 1989;140: 157-65.

7 Setzer ES, Webb IB, Wassenaar JW, Reeder JD, Mehta PS, Eitzman DV. Platelet dysfunction and coagulopathy in intraventricular hemorrhage in the premature infant. f Pediatr 1982;100:599-605.

8 Beverley DW, Chance GW, Inwood MJ, Schaus $M$, O'Keefe, B. Intraventricular haemorrhage and haemostasis defects. Arch Dis Child 1984;59:444-8. 9 McDonald MM, Johnson, ML, Rumack CM, et al. Role of coagulopathy in newborn intracranial hemorrhage. Pediatrics $1985 ; 74: 26-31$.

10 Amato M, Fauchere JC, Hermann U. Coagulation abnormalities in low birth weight infants with peri-intraventricular hemorrhage. Neuropediatrics 1988;19:154-7.

11 Horbar JD. Prevention of periventricular-intraventricular hemorrhage. In: Sinclair JC, Bracken M, eds. Effective care of the newborm infant. Oxford: Oxford University Press, 1992;562-89. 